\title{
A Case Study of Team-Based Learning for Evolutionary Biology Classes in Biology Majors
}

\author{
Paulo Oliveira1, Diogo Figueiredo $^{2}$ \\ University of Évora, Biology Department Portugal ${ }^{1,2}$
}

\begin{abstract}
The authors describe the experience with a partial implementation of Team-Based Learning strategy, as was carried out in the context of the Evolutionary Biology course, an obligate part of the undergraduate Biology curriculum at the University of Évora. In contrast to previous attempts at turning the students attending this course from their notorious inertia, this strategy finally accomplished a significant unfolding of the students' immediate interest and thence helped them attain a richer learning experience. The recommendations by the developers of Team-Based Learning served as basis for this implementation, which is laid out in protocol format for the present article and illustrated with examples from the themes proposed. The results from a survey on this implementation of Team-Based Learning, made available to the students, gave an important feedback for discussion of the strengths and weaknesses in the experience so far. Although this implementation arguably fulfills most of the core elements of the recommended Team-Based Learning procedure, considerations are made on the possible need to upgrade to a full implementation of Team-Based Learning in this course.
\end{abstract}

Keyword: Active learning, Biology teaching, Evolutionary Biology, Team-Based Learning. 